\title{
Impact of large island perturbations on turbulent blob transport in tokamaks
}

D. Reiser

Citation: Physics of Plasmas 14, 082314 (2007); doi: 10.1063/1.2771133

View online: https://doi.org/10.1063/1.2771133

View Table of Contents: http://aip.scitation.org/toc/php/14/8

Published by the American Institute of Physics

\section{Articles you may be interested in}

Dynamics of ion temperature gradient turbulence and transport with a static magnetic island

Physics of Plasmas 23, 022304 (2016); 10.1063/1.4941704

Shrinking of core neoclassical tearing mode magnetic islands due to edge localized modes and the role of ionscale turbulence in island recovery in DIII-D

Physics of Plasmas 24, 062503 (2017); 10.1063/1.4985078

Increased electron temperature turbulence during suppression of edge localized mode by resonant magnetic perturbations in the DIII-D tokamak

Physics of Plasmas 24, 112305 (2017); 10.1063/1.4999785

A model for generation of high wavenumber fluctuations by external magnetic field perturbations in edge pedestal plasmas

Physics of Plasmas 24, 012507 (2017); 10.1063/1.4974166

Flow shear induced fluctuation suppression in finite aspect ratio shaped tokamak plasma

Physics of Plasmas 2, 1648 (1995); 10.1063/1.871313

Impact of resonant magnetic perturbations on nonlinearly driven modes in drift-wave turbulence

Physics of Plasmas 19, 055903 (2012); 10.1063/1.3694675

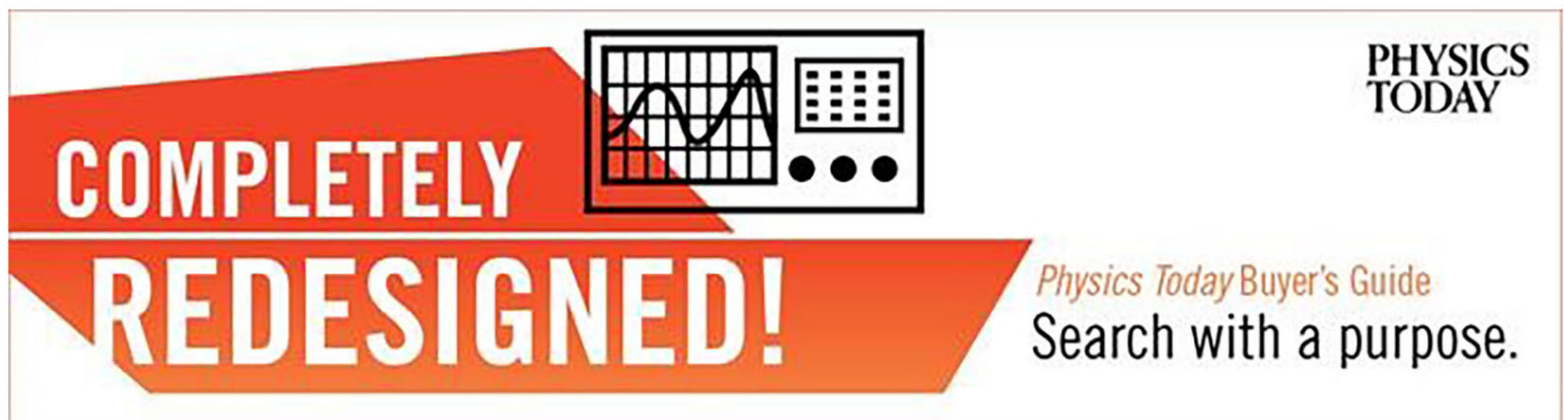




\title{
Impact of large island perturbations on turbulent blob transport in tokamaks
}

\author{
D. Reiser \\ Institut für Energieforschung-Plasmaphysik, Forschungszentrum Jülich GmbH, EURATOM Association, \\ Trilateral Euregio Cluster, D-52425 Jülich, Germany
}

(Received 9 May 2007; accepted 20 July 2007; published online 29 August 2007)

\begin{abstract}
The turbulent blob transport in the tokamak edge and its structural changes in perturbed magnetic configurations like islands and ergodic structures is studied by means of numerical simulations. An explicit finite-difference algorithm for treating three-dimensional plasma fluid turbulence models is presented and described in detail. For an unperturbed magnetic equilibrium the numerical results show an appearance of density blobs in the vicinity of the last closed flux surface. In the presence of an ergodic magnetic perturbation strong suppression of blob amplitudes and local reversal of the radial $E \times B$-flux close to the resonances is observed. (C) 2007 American Institute of Physics.
\end{abstract}

[DOI: $10.1063 / 1.2771133$ ]

\section{INTRODUCTION}

Numerical simulations of drift wave turbulence are a basic tool for the theoretical understanding of plasma dynamics in tokamaks. The interest in three-dimensional turbulence simulations including complex magnetic topologies like open field lines, islands, and ergodic structures have been stimulated further by recent observations at DIII-D, ${ }^{1,2} \mathrm{JET}^{3}$ and at TEXTOR-DED. ${ }^{4,5}$ There it has been proved that resonant magnetic perturbation fields have a beneficial effect for the control of bursty particle and heat transport. Typically the radial transport of particles and heat in the tokamak scrapeoff layer (SOL) is not smooth and steady but rather exhibits an intermittent, bursty behavior which occurs due to the presence of the last closed flux surface (LCFS), i.e., a boundary where an abrupt change from closed to open magnetic field lines occurs. In the above mentioned experiments an externally induced magnetic perturbation field has been applied to change the topology by resonant structures, i.e. magnetic islands. It is found that these ergodized magnetic structures cause a strong suppression of blob transport in the SOL and that edge localized modes (ELM's) are reduced or even disappear. A number of numerical investigations of SOL turbulence and blob transport have been performed on the basis of 2D fluid models ${ }^{6-9}$ and 3D gyrofluid models. ${ }^{10}$ Simulations of 3D drift turbulence in ergodized magnetic configurations without SOL effects have been reported in Refs. 11 and 12. In this paper we consider both open (SOL-region) and closed field lines (edge-region) and take into account the presence of large magnetic islands too. We use a 3D fluid drift turbulence model to study blob formation in the SOL of tokamaks and its significant changes due to the impact of a strong change in magnetic topology. The fluid model employed and our numerical approach are similar to those of previous studies using the codes TYR (Ref. 13) and DALF3. ${ }^{14,15}$ However several different features in the numerical approach, in particular the treatment of geometry, are noteworthy, therefore we describe certain aspects in detail. It is shown that experimentally observed phenomena like amplitude reduction of the intermittent particle fluctuations and local $E \times B$-fluxes can be reproduced qualitatively by numerical simulations for TEXTOR-like conditions.

This paper is organized as follows: In Sec. II the basic four field fluid model is presented in its general form and the simplifications leading to the local form used in this work is elucidated. For the numerical solution of the model equations the code ATTEMPT (A Three-dimensional Tracer for ElectroMagnetic Plasma Turbulence) has been developed and its algorithmic structure and the implementation of boundary conditions is outlined in Secs. III and IV. Simulation results for flux driven turbulence in the edge-SOL region are presented in Sec. $\mathrm{V}$, demonstrating the appearance of blob transport in an unperturbed magnetic equilibrium due to the presence of a LCFS. The extension of the computations by the inclusion of an ergodized magnetic perturbation field shows a suppression of the blob transport. Time traces of the intermittent particle transport bear resemblance to the experimentally observed ones. The $E \times B$-flux is strongly changed by the magnetic perturbation. An alternating pattern with negative and positive signs corresponding to the symmetry of the perturbation field dominates the static contribution to the $E \times B$-flux, whereas the turbulent flux is reduced around the O-points. These structures hinder the density blobs from their radial movement. Finally a summary and a discussion are given in Sec. VI followed by concluding remarks in Sec. VII.

\section{A FOUR FIELD MODEL FOR PLASMA TURBULENCE}

In this section the basic features of the fluid turbulence model underlying our numerical investigations are outlined. Starting from a general set of two fluid equations in drift ordering the local model is introduced by partial linearization. Details on the choice of suitable coordinates and scalings to obtain a dimensionless coordinate-dependent representation of the local model have been put into Appendices A-C.

Models for plasma fluid turbulence are often based on Braginskii two fluid equations combined with Maxwell's equations under drift ordering, i.e., approximate solution of 
the perpendicular momentum equation and neglect of parallel compared to perpendicular derivatives (except in the parallel gradient $\left.\boldsymbol{\nabla}_{\|}\right) .{ }^{16-19}$ By neglecting temperature dynamics, considering cold ions (ion temperature $T_{i}=0$ ) and assuming a constant electron temperature $T_{e}$ one obtains the four-field version of the dissipative drift-Alfvén model ${ }^{20}$ describing the nonlinear evolution of small scale dynamics of the electrostatic potential $\phi$, the density $n$, the parallel magnetic potential $A$, and the parallel ion velocity $u$,

$$
\begin{aligned}
& \frac{d n}{d t}=\frac{1}{e} \nabla_{\|} J-n \nabla_{\|} u-n \mathcal{K}(\phi)+\frac{T_{e}}{e} \mathcal{K}(n), \\
& \frac{n m_{i}}{B_{0}^{2}} \frac{d w}{d t}=\nabla_{\|} J+T_{e} \mathcal{K}(n), \\
& \frac{\partial A}{\partial t}+\frac{m_{e}}{e^{2} n} \frac{d J}{d t}=\frac{T_{e}}{e n} \nabla_{\|} n-\nabla_{\|} \phi-\eta_{\|} J, \\
& n m_{i} \frac{d u}{d t}=-T_{e} \nabla_{\|} n,
\end{aligned}
$$

with the auxiliary relations

$$
w=\nabla_{\perp}^{2} \phi ; \quad \mu_{0} J=-\nabla_{\perp}^{2} A
$$

and operators defined by

$$
\begin{aligned}
& \frac{d}{d t}=\frac{\partial}{\partial t}+\mathbf{v}_{E} \cdot \boldsymbol{\nabla}, \\
& \mathbf{v}_{E} \cdot \boldsymbol{\nabla} f=\frac{\mathbf{B}}{B^{2}} \cdot(\boldsymbol{\nabla} \phi \times \nabla f), \\
& \mathcal{K}(f)=\boldsymbol{\nabla} \cdot\left(\frac{\mathbf{B} \times \boldsymbol{\nabla} f}{B^{2}}\right), \\
& \boldsymbol{\nabla}_{\|} f=\frac{\mathbf{B}}{B} \cdot \boldsymbol{\nabla} f-\frac{\mathbf{B}}{B^{2}} \cdot(\boldsymbol{\nabla} A \times \nabla f), \\
& \nabla_{\perp}^{2}=\nabla^{2} f-\boldsymbol{\nabla} \cdot \frac{\mathbf{B}}{B} \frac{\mathbf{B}}{B} \cdot \nabla f .
\end{aligned}
$$

Here B denotes the equilibrium magnetic field. Thus the model Eqs. (1)-(4) consists of the equation of continuity, the quasineutrality condition $\boldsymbol{\nabla} \cdot \mathbf{J}=0$, Ohm's law, and the total momentum balance, respectively. Here $\eta_{\|}$is the classical parallel resistivity, ${ }^{16} m_{e}$ and $m_{i}$ are the electron and ion mass, respectively, and $n$ is the particle density $\left(n=n_{e}=n_{i}\right)$. Note that parallel advection has been neglected. The global model Eqs. (1)-(4) can be simplified further by the neglect of all nonlinearities except for the action of $E \times B$ advection and magnetic flutter. This is done by setting constant the density $n$ and keeping its profile in the temporal and spatial derivatives only. A toroidal magnetic equilibrium field is assumed as defined by Eq. (A16) and the set of field aligned coordinates defined by Eqs. (A20) and (A21) is employed in the limit of small $\epsilon=r / R_{0}$ to obtain an explicit form of the model equations. Finally a proper scaling as specified by Eqs.
(C1)-(C5) gives the following dimensionless form of the local model:

$$
\begin{aligned}
& \frac{\partial n}{\partial t}=\{\phi, n\}-\mathcal{K}(\phi-n)+\nabla_{\|}(J-u), \\
& \frac{\partial w}{\partial t}=-\{\phi, w\}+\mathcal{K}(n)+\nabla_{\|}, \\
& \hat{\beta} \frac{\partial A}{\partial t}+\hat{\mu} \frac{\partial J}{\partial t}=-\hat{\mu}\{\phi, J\}+\nabla_{\|}(n-\phi)-\hat{C} J, \\
& \hat{\epsilon} \frac{\partial u}{\partial t}=-\hat{\epsilon}\{\phi, u\}-\nabla_{\|} n,
\end{aligned}
$$

where

$$
w=\nabla_{\perp}^{2} \phi ; \quad J=-\nabla_{\perp}^{2} A .
$$

The remaining dimensionless parameters of the model are considered as constants in the local model. According to the discussion of the coordinate systems $(s, x, y)$ and $(\sigma, \chi, \sigma)$ in Appendices A-C the scaled operators for small $\epsilon=r / R_{0}$ are given by

$$
\begin{aligned}
& \{f, g\}=\frac{\partial f}{\partial \chi} \frac{\partial g}{\partial \eta}-\frac{\partial f}{\partial \eta} \frac{\partial g}{\partial \chi}, \\
& \mathcal{K}(f)=-\omega_{B}\left(\cos s \frac{\partial f}{\partial \eta}+\sin s \frac{\partial f}{\partial \chi}\right), \\
& \nabla_{\|} f=\frac{\partial f}{\partial s}-\hat{\beta}\{A, f\}, \\
& \nabla_{\perp}^{2} f=\frac{\partial^{2} f}{\partial \chi^{2}}+\frac{\partial^{2} f}{\partial \eta^{2}} .
\end{aligned}
$$

Note that a mixed representation is used in this formulation, i.e., $s, \chi$, and $\eta$ do not constitute a single coordinate system; they belong to different coordinate systems. Equations (11)-(19) form the basis for the numerical studies in this paper. Apart from the neglect of dissipation and extra instabilities due to fluctuations in the temperature this model forms quite a general set including (linear) physics such as sound waves, drift Alfvén waves, and drift resistive ballooning modes. The inclusion of the magnetic flutter and the induction term in Ohm's law make it an electromagnetic model. A more detailed discussion of the basic physics described by the model can be found in Ref. 20, and references therein. Finally it is to be noted that Eqs. (1)-(5) or Eqs. (11)-(15) do not represent a complete model for plasma turbulence insofar that they do not include dissipation effects acting on scales smaller than the drift scale $\rho_{s}$. Without such a dissipation mechanism the turbulence cannot saturate, because the resistivity $\hat{C}$ is not strong enough to compensate for the turbulent drive. Such a sink for turbulent energy has to be included by hand to mimic the small scale kinetic processes of a real physical system. Here we introduce dissipative processes by the extension 


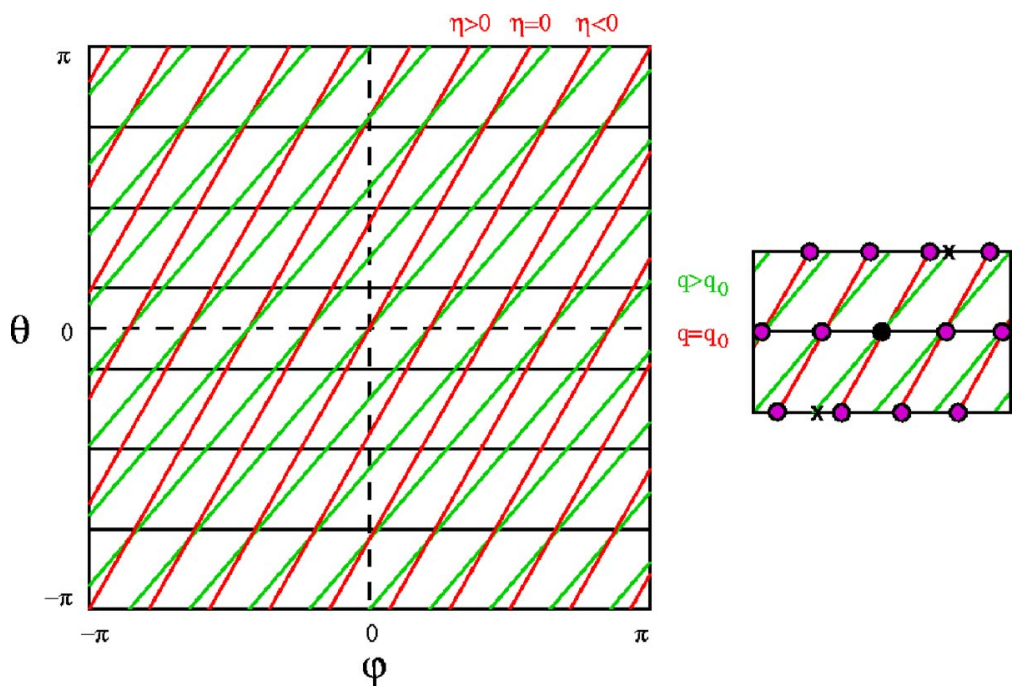

FIG. 1. (Color online) Illustration of the shift in the $\eta$-coordinate and the need for interpolation in Eq. (23). The red lines are $\sigma$-coordinate lines. At $q=q_{0}$ the coordinate lines of $s$ and $\sigma$ are identical. The black horizontal lines are the coordinate lines of $y$ and $\eta$. For $q$ $\neq q_{0}$ a certain point on an $s$-coordinate line (green) is in general located between the grid points of the $(\sigma, \eta)$-grid. In the zoomed picture on the right the points needed for evaluation of the parallel derivative at the midpoint (black circle) are labeled by crosses. The magenta colored circles are the grid points of the $(\sigma, \eta)$-grid used in the numerical scheme.

$$
\frac{\partial f}{\partial t}=S+\mu_{\perp} D_{\perp}+\mu_{\|} D_{\|},
$$

where $f$ is $n, w$ or $u$ and $S$ is the respective rhs of Eqs. (11)-(14). The additional dissipation terms are chosen as $D_{\perp}=\nabla_{\perp}^{4} f$ and $D_{\|}=\nabla_{\|}^{2} f$. However the choice of the dissipation coefficients $\mu_{\perp}$ and $\mu_{\|}$is a subtle problem, because they should be in a range that they provide sufficient dissipation but do not strongly disturb the basic driving mechanisms for turbulence.

\section{NUMERICAL ALGORITHM}

For the purpose of a numerical solution of Eqs. (11)-(15) the ATTEMPT code has been developed. The numerical approach implemented has many similarities to previously used schemes. ${ }^{13-15}$ But for the reason of a self-contained description and due the particular differences in the treatment of field aligned coordinates details of the ATTEMPT code are sketched with special emphasis on the evaluation of parallel gradients.

Employing a finite difference approach, any dependent variable $f$ is discretized on a $N_{\sigma} \times N_{\chi} \times N_{\eta}$-grid in the $\sigma$ - $\chi$ - $\eta$-domain, i.e., $f_{i, j, k}=f\left(\sigma_{i}, \chi_{j}, \eta_{k}\right)$. The grid dimensions in scaled units are

$$
L_{\sigma}=2 \pi ; \quad L_{\chi}=\frac{N_{\chi}}{N_{\eta}} \frac{L_{\eta}}{\pi \hat{s}} ; \quad L_{\eta}=\frac{2 \pi r_{0}}{q_{0} \rho_{s} N_{f}},
$$

where the integer number $N_{f}$ represents the number of (identical) flux tubes in the toroidal annulus such that $N_{f}=1$ means that we are dealing with a flux tube that covers the complete flux surface. The magnetic shear $\hat{s}$ is assumed to be constant, describing a linear profile $q=q_{0}\left(1+\hat{s} \chi \rho_{s} / r_{0}\right)$ of the equilibrium magnetic field in the computational domain [cf. Eq. (A16)]. The strategy for the numerical solution is to discretize the rhs of Eqs. (11)-(14), i.e. $\partial f /\left.\partial t\right|_{i, j, k}=F_{i, j, k}$, and to use the third order stiffly stable scheme of Karniadakis for time stepping ${ }^{21}$

$$
\begin{aligned}
f_{i, j, k}^{t+\Delta t}= & \frac{6}{11}\left[3 f_{i, j, k}^{t}-\frac{3}{2} f_{i, j, k}^{t-1}+\frac{1}{3} f_{i, j, k}^{t-2}+\Delta t\left(3 F_{i, j, k}^{t}-3 F_{i, j, k}^{t-1}\right.\right. \\
& \left.\left.+F_{i, j, k}^{t-2}\right)\right] .
\end{aligned}
$$

The linear curvature terms of the form $\mathcal{K}(f)$ are discretized by standard second order centered differences, but the nonlinear Poisson bracket $\{f, g\}$ is evaluated according to the Arakawa scheme. ${ }^{22}$ The solution of Helmholtz equations to compute $A, J$, and $\phi$ is done by means of a Fourier decomposition in the periodic coordinate $\eta$ and subsequent solution of a tridiagonal system in $\chi$. The parallel derivative $\partial f / \partial s$ is evaluated by a second order discretization

$$
\left.\frac{\partial f}{\partial s}\right|_{i, j, k}=\frac{f\left(\sigma_{i+1}, \chi_{j}, \eta_{k}+\delta_{j}\right)-f\left(\sigma_{i-1}, \chi_{j}, \eta_{k}-\delta_{j}\right)}{2 \Delta \sigma},
$$

where $\delta_{j}=-\hat{s} \chi_{j} \Delta \sigma$. The shift $\delta_{j}$ is a consequence of the relations

$$
\frac{\partial \sigma}{\partial s}=1 ; \quad \frac{\partial \chi}{\partial s}=0 ; \quad \frac{\partial \eta}{\partial s}=\frac{r_{0}}{\rho_{s}} \frac{q_{0}-q}{q_{0}}
$$

following for $\epsilon \ll 1$ from Eqs. (A10) and (A11) (see also Fig. 1). The centered difference in Eq. (23) is computed via Fourier interpolation in the periodic coordinate $\eta$, i.e. using

$$
\begin{aligned}
& f\left(\sigma_{m}, \chi_{j}, \eta\right)=\sum_{l} \bar{f}_{m, j, l} e^{i k_{l} \eta}, \\
& \bar{f}_{m, j, l}=\frac{1}{N_{\eta}} \sum_{k} f\left(\sigma_{m}, \chi_{j}, \eta_{k}\right) e^{-i k_{l} \eta_{k}}
\end{aligned}
$$

with wave numbers $k_{l}=2 \pi l / L_{\eta}$, and $l$ in the range $-N_{\eta} / 2$ $\leq l \leq N_{\eta} / 2-1$. This gives

$$
\left.\frac{\partial f}{\partial s}\right|_{m, j, k}=\sum_{l} \frac{\bar{f}_{m+1, j, l} e^{i k_{l} \delta_{j}}-\bar{f}_{m-1, j, l} e^{-i k_{l} \delta_{j}}}{2 \Delta \sigma} e^{i k_{l} \eta_{k}} .
$$




\section{BOUNDARY CONDITIONS}

The boundary conditions for the model calculations are implemented by the use of guard cells, i.e., additional cells at the edges of each grid dimension (here labeled by the indices 0 and $N_{\nu}+1$, where $\left.\nu=\sigma, \chi, \eta\right)$. For the $\eta$-direction periodic boundary conditions are used and the guard cells are filled for each scalar field by $f\left(\sigma_{i}, \chi_{j}, \eta_{k}\right)=f\left(\sigma_{i}, \chi_{j}, \eta_{k+N}\right)$. For the $\chi$-domain zero Dirichlet boundary conditions are used for all scalar fields, except the density $n$, i.e.,

$$
f\left(\sigma_{i}, \chi_{0}, \eta_{k}\right)=f\left(\sigma_{i}, \chi_{N_{\chi^{+1}}}, \eta_{k}\right)=0 .
$$

The boundary conditions for the density are different at the two edges. For the outer boundary again zero Dirichlet boundary conditions are used, but at the inner boundary Neumann conditions are imposed to prescribe a constant influx of particles (flux driven turbulence). This is done by

$$
n\left(\sigma_{i}, \chi_{0}, \eta_{k}\right)=\left.\langle n\rangle\right|_{\chi_{1}}-\left.\Delta \chi \frac{\partial\langle n\rangle}{\partial \chi}\right|_{\chi_{0}},
$$

where $\langle n\rangle$ denotes the axisymmetric $\sigma-\eta$-average of the density and $\left.\partial_{\chi}\langle n\rangle\right|_{\chi_{0}}$ is the desired gradient of $\langle n\rangle$ at the boundary. Note that this choice prescribes the gradient of the axisymmetric part only, whereas the nonaxisymmetric part fulfills again zero Dirichlet boundary conditions. For the $\sigma$-domain the boundary conditions are guided by the relation

$$
f(\sigma, \eta)=f\left(\sigma \pm 2 \pi, \eta \pm q_{0} N_{f} L_{\eta}\right)=f\left(\sigma, \eta \pm L_{\eta}\right) .
$$

This relation results from the requirement of the global periodicity constraint in the poloidal angle $\theta$ and the toroidal angle $\varphi$, respectively. ${ }^{23}$ Thus one finds for the guard cells for each scalar field

$$
\begin{aligned}
& f\left(\sigma_{0}, \chi_{j}, \eta_{k}\right)=f\left(\sigma_{N_{\sigma}}, \chi_{j}, \eta_{k}+N_{f} q_{0} L_{\eta}\right), \\
& f\left(\sigma_{N_{\sigma}+1}, \chi_{j}, \eta_{k}\right)=f\left(\sigma_{1}, \chi_{j}, \eta_{k}-N_{f} q_{0} L_{\eta}\right) .
\end{aligned}
$$

If $q_{0}$ is not an integer number this needs an interpolation like the one used in the evaluation of parallel derivatives elucidated in the last section. If $q_{0}$ is an integer the relations reduce to simple periodic boundary conditions for the $\sigma$-coordinate.

The implementation of a SOL into the computational domain is illustrated for a toroidal line limiter located at the edges of the $\sigma$-domain (in this work always at $\sigma= \pm \pi$, representing a limiter at the high field side). Following Ref. 10, we employ the model

$$
\frac{\partial n}{\partial s}=0, \quad \frac{\partial \phi}{\partial s}=0, \quad u= \pm \frac{n}{\sqrt{\hat{\epsilon}}}, \quad J= \pm \frac{\phi-\Lambda n}{\sqrt{\hat{\epsilon}}},
$$

where

$$
\Lambda=\frac{1}{2} \ln \left(\frac{m_{i}}{2 \pi m_{e}}\right)
$$

to prescribe the conditions at the limiter plates. According to Eq. (23) the implementation for the fields $n$ and $\phi$ is done by

$$
\begin{aligned}
& f\left(\sigma_{0}, \chi_{j}, \eta_{k}\right)=f\left(\sigma_{1}, \chi_{j}, \eta_{k}-\delta_{j}\right), \\
& f\left(\sigma_{N_{\sigma^{+1}}}, \chi_{j}, \eta_{k}\right)=f\left(\sigma_{N_{\sigma}}, \chi_{j}, \eta_{k}+\delta_{j}\right) .
\end{aligned}
$$

For the velocity $u$ we use

$$
\begin{aligned}
& u\left(\sigma_{0}, \chi_{j}, \eta_{k}\right)=-u\left(\sigma_{1}, \chi_{j}, \eta_{k}-\delta_{j}\right)-\frac{2}{\sqrt{\hat{\epsilon}}} n\left(\sigma_{1}, \chi_{j}, \eta_{k}\right), \\
& u\left(\sigma_{N_{\sigma^{+}}+}, \chi_{j}, \eta_{k}\right)=-u\left(\sigma_{N_{\sigma}}, \chi_{j}, \eta_{k}+\delta_{j}\right)+\frac{2}{\sqrt{\hat{\epsilon}}} n\left(\sigma_{N_{\sigma}}, \chi_{j}, \eta_{k}\right)
\end{aligned}
$$

and a similar construction for the current density $J$ according to the boundary values of Eq. (32).

\section{RESULTS ON FLUX DRIVEN EDGE-SOL TURBULENCE AND IMPACT OF MAGNETIC PERTURBATIONS}

In this section we present simulation results for a computational domain and parameters chosen in order to be fairly close to conditions typical for L-mode (low confinement mode) TEXTOR-DED discharges. Two scenarios are considered: first the flux driven turbulent dynamics in the vicinity of the LCFS with a background magnetic field prescribed by the unperturbed equilibrium Eq. (A16), second the identical situation but with a modified background field including resonant magnetic perturbations. The perturbation field is included by the replacement $A \rightarrow A+A *$ in the model equations, ${ }^{11}$ where

$$
A_{*}=\sum_{m_{*}=-1}^{1} A_{m_{*}} e^{n_{*} k_{\eta} \chi} \cos \left(m_{*} \sigma+n_{*} k_{\eta} \eta\right)
$$

with toroidal mode number $n_{*}=4$ and $k_{\eta}=2 \pi / L_{\eta}$, representing a static externally induced perturbation field consisting of the three modes with poloidal/toroidal mode numbers 11/4, 12/4, and 13/4 (called Ergodic Divertor regime, ED). In the unperturbed case it is found that density blobs are formed at the LCFS and move radially outwards. The flux surface averaged density profile is flattened in the SOL-region due to strong parallel flows of particles to the limiter plates being located at the high field side. The perturbations in the magnetic configuration gives rise to a significant suppression of the observed amplitudes of the intermittent particle transport close to the LCFS. Also the radial $E \times B$-flux is altered substantially and the corresponding movement of particle blobs is impeded. A resonant structure appears where regions of positive flows alternates with regions of negative flows. However the flux surface averaged net transport in radial direction is significantly reduced. In detail the setup of the computations is as follows. We consider the entire flux surfaces in the range $2 \leq q \leq 4$ around the reference radius $r_{0}=0.35 \mathrm{~m}$, i.e., $0.29 \mathrm{~m} \leq r \leq 0.41 \mathrm{~m}$. The dimensionless model parameters are chosen as $\omega_{B}=0.046, \hat{C}=0.96, \hat{\beta}=6.9$, $\hat{\mu}=4.7, \hat{\epsilon}=17227$, and $\hat{s}=2$. This represents a plasma with physical parameters $R_{0}=1.75 \mathrm{~m}, m_{i}=2 m_{p}, q_{0}=3, B_{0}=1 \mathrm{~T}$, $L_{\perp}=4 \cdot 10^{-2} \mathrm{~m}, n_{0}=2 \cdot 10^{19} \mathrm{~m}^{-3}$ and $T_{e}=100 \mathrm{eV}$. The number of grid points are $N_{\sigma} \times N_{\chi} \times N_{\eta}=16 \times 128 \times 128$ giving a resolution of about $1 \rho_{s}$ in the $\chi$-direction and $4 \rho_{s}$ in the 

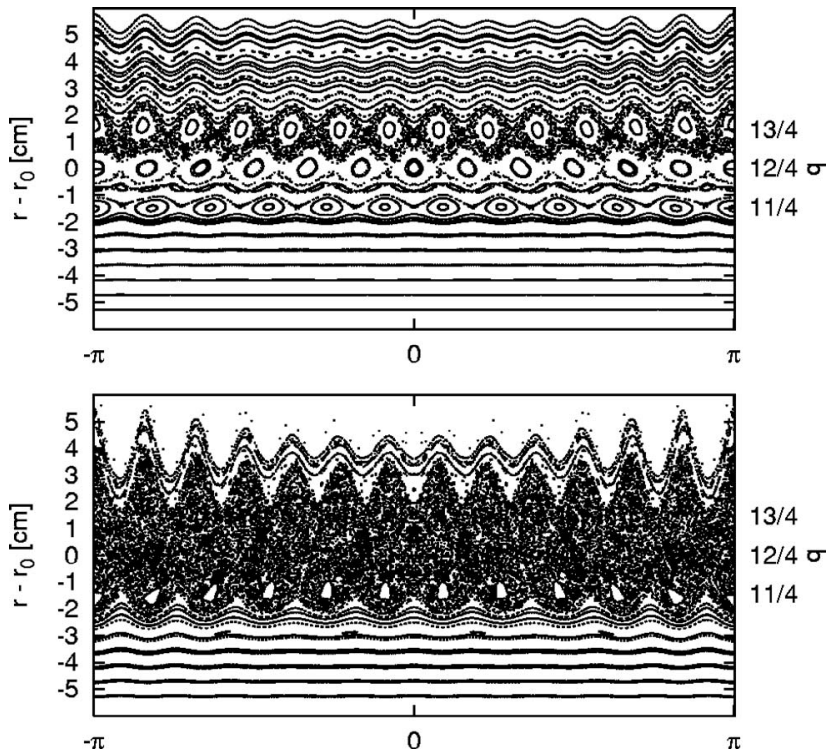

FIG. 2. Poincaré-plot of the magnetic field with magnetic perturbation. Top: For a perturbation strength of one fourth of that used in the computation. Bottom: For the perturbation field used in the simulations.

$\eta$-direction. The gradient of the axisymmetric part of the density at the inner boundary in the $\chi$-direction is prescribed by $\left.\partial_{\chi}\langle n\rangle\right|_{\chi_{0}}=-5$ and the dissipation parameters are chosen as $\mu_{\perp}=\mu_{\|}=0.01$. The time step is $\Delta t=0.03 L_{\perp} / c_{s}$ corresponding to $1.73 \cdot 10^{-8} \mathrm{~s}$. The SOL boundary conditions of Eq. (32) are used for the outer half of the radial domain, i.e., for $r>r_{0}$. The simulation is started with a linear density profile inside the LCFS and zero in the SOL, and is followed until the total particle content is statistically stationary indicating that the turbulent drive by the density gradient is also statistically stationary. This is done by tracking the total particle content $N=\int n d V$ of the computational volume until the standard deviation of its temporal average $\bar{N}$ is less than the standard deviation of $N$ itself. In practice this means that the particle content (and connected with this always also the axisymmetric density profile) shows a clear fluctuation around a "stable" (slightly varying) mean value when inspected with the naked eye. For the parameters used here this state is reached after a simulation time of about $1.5 \cdot 10^{4} L_{\perp} / c_{S}$, corresponding to $8.7 \mathrm{~ms}$. Afterwards the simulation is continued for additional $10^{5}$ time steps for diagnosis purposes. A second simulation run starting with the same profiles is performed keeping all parameters unchanged but including the magnetic perturbations with $A_{-1}=1.2 \cdot 10^{-5} \mathrm{Tm}$, $A_{0}=-1.2 \cdot 10^{-5} \mathrm{Tm}$, and $A_{+1}=1.2 \cdot 10^{-5} \mathrm{Tm}$. This gives a maximum radial magnetic field of about $1.2 \cdot 10^{-3} \mathrm{~T}$ at $r=r_{0}$ and a localization at the high field side. The Poincaré-plot in Fig. 2, bottom, illustrates the resulting total (vacuum) magnetic field in the computational domain. The three island chains strongly overlap causing a considerable amount of ergodization. The final flux surface and time averaged profiles of density $\langle\bar{n}\rangle$ and radial $E \times B$-flow $\langle\bar{\Gamma}\rangle$, where $\Gamma=n \partial_{\eta} \phi$, are shown in Fig. 3. Here the bar denotes a time average taken over the $10^{5}$ time steps of the diagnosis run. The magnetic perturbations give rise to a flattening of the density profile and a reduction of the $E \times B$-flux outside the LCFS. The same data are illustrated also by Fig. 4 showing the density gradient length $L$ and the effective diffusion coefficient $D$ defined by $\partial_{r}\langle\bar{n}\rangle=\langle\bar{n}\rangle / L=\langle\bar{\Gamma}\rangle / D$. The latter represents the radial diffusion coefficient which would be necessary to provide a radial diffusive flux of the same magnitude as the net $E \times B$-flux computed. This quantity is often used to transfer results of turbulence models into transport models where the turbulent radial transport is approximated by a diffusive process. The gradient length $L$ is increased by a factor of about 2 in the vicinity of the LCFS and the effective diffusion coefficient $D$ is found to be in the typical order of magnitude of about $1 \mathrm{~m}^{2} / \mathrm{s}$ and is increased inside the LCFS and decreased outside. Of course the magnetic perturbation field introduces a strong poloidal variation of the radial transport. This is demonstrated by the time averaged contour plots of $\bar{\Gamma}$ in Figs. 6 and 7 below. Time averaged spectra of the density are shown in Fig. 5 for the region inside the LCFS (closed field lines) and outside (open field lines), respectively. The spectra for the region $r<r_{0}$ are almost unchanged while the spectra for $r>r_{0}$ are very different. The magnetic perturbation field induces resonances at the harmonics $n_{*}$ $=4,8,12$, etc. of the basic toroidal mode of the perturbation. This effect is typical in the presence of resonant magnetic fields as discussed in Ref. 11. Due to the presence of a SOL region, in the unperturbed configuration particle blobs (elongated streamers) are formed in the vicinity of the LCFS and move radially outwards. This is illustrated by the snapshot of density $n$ shown in Fig. 6 (top) showing the $\theta-r$-profile in the poloidal cut at $\varphi=0$. The blobs are extended about $1-$ $2 \mathrm{~cm}$ into SOL-region. In the scenario with induced magnetic perturbation these extended blobs disappear as shown in the corresponding plot of Fig. 7. Also shown in Fig. 6 is
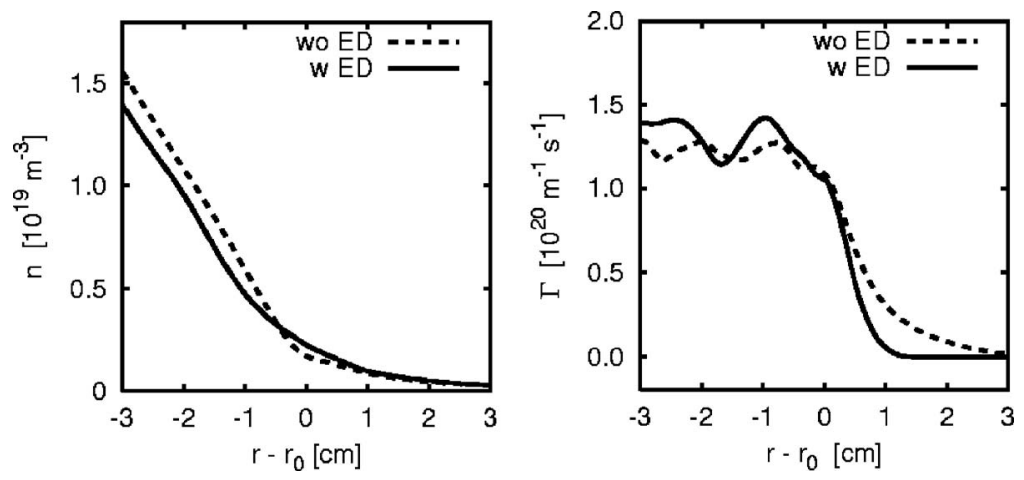

FIG. 3. Profiles of density $\langle\bar{n}\rangle$ and $E \times B$-flux $\langle\bar{\Gamma}\rangle$ for flux driven scenarios with and without magnetic perturbation. 

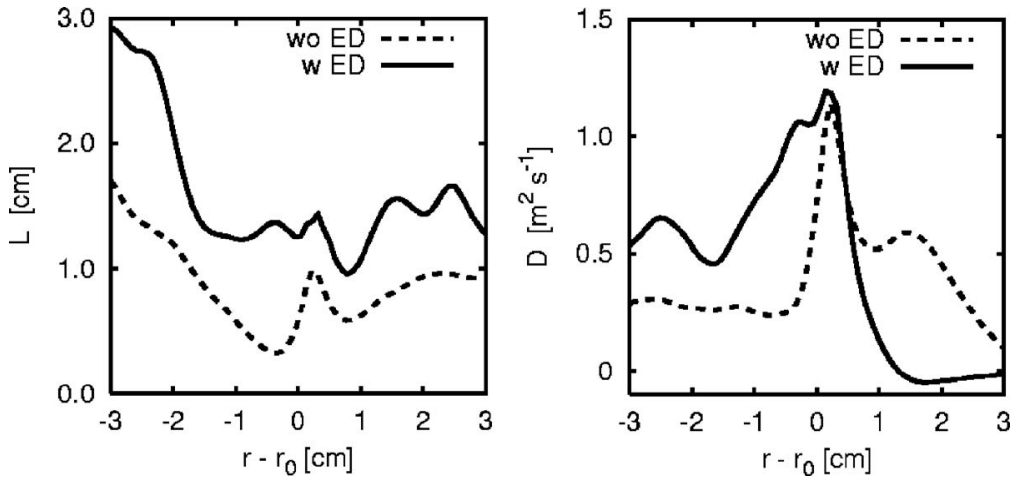

FIG. 4. Profiles of density gradient length $L$ and diffusion coefficient $D$ for flux driven scenarios with and without magnetic perturbation. the time averaged flux $\bar{\Gamma}$ in the same poloidal cut. The flux is positive almost everywhere and does not show any pronounced symmetry. But as can be seen in Fig. 7 the perturbation field induces a pattern of flows where the sign is clearly alternating corresponding to the symmetry of the magnetic perturbation. To study the intermittent behavior also "numerical probes" have been used to trace the density fluctuations and $E \times B$-fluxes at different radial positions close to the outer midplane (namely at $\theta=-2 \pi / 16$ and $\varphi=$ $-2 \pi / 128)$. Examples of such time traces are shown in Figs. 8 and 9 for the radial locations $r=r_{0}$ and $r=r_{0} \pm 1 \mathrm{~cm}$. They demonstrate that the local fluctuations of plasma density are reduced in the presence of the magnetic perturbation and that the $E \times B$-flux on the one hand is reduced in magnitude and on the other hand its temporal average changes sign. To get more insight into the quantitative modifications of the turbulent fluctuations the frequency spectra corresponding to the numerical probe signals of Figs. 8 and 9 are depicted in Fig. 10. All graphs show a reduction of density and $E \times B$-flux fluctuations due to the magnetic perturbation by a factor of 2-4 for the low frequency part $(<10 \mathrm{kHz})$ of the spectrum, in particular for the probes at the LCFS and in the SOL. Another observation is that the peak in the frequency spectrum for the fluctuations inside the LCFS, which can be attributed to geodesic acoustic modes (GAM) activity with frequency $\nu_{\mathrm{GAM}} \approx \sqrt{2+1 / q^{2}} c_{s} / 2 \pi R_{0}$ in the unperturbed scenario, ${ }^{24}$ is shifted towards higher frequencies in the presence of the perturbation field. Finally Fig. 11 shows timeaveraged $\theta$-r-profiles of the density $n$, the electric potential $\phi$, the nonadiabatic part of electron density $h=n-\phi,{ }^{18}$ and the
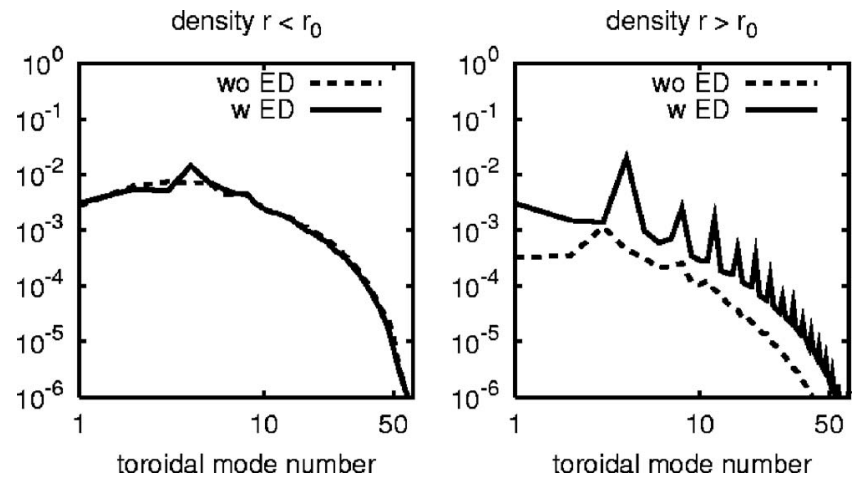

FIG. 5. Spectra of density $\bar{n}$ with and without magnetic perturbation for the regions of closed and open field lines separately.
$E \times B$-flux $\Gamma$, for the case with magnetic perturbation. In order to detect to what extent the symmetry of the perturbation can be rediscovered in the simulation results the $\mathrm{X}$ - and O-points of the three island chains taken into account are labeled in the plots. It can be seen that in particular $\bar{h}$ shows a clear correspondence to the symmetry of the magnetic field. Its minima around $r=r_{0}$ are manifestly located around the O-points of the 12/4-mode contribution, and the resulting pattern is very similar to the island structure of Fig. 2 (top), representing a four times lower magnetic perturbation than the one used in the simulation. This appearance of residual island structures in the profiles of $n, \phi$ and in particular $h$ shown in Fig. 11 might be surprising if one compares these structures with the Poincaré-plot of the magnetic perturbation field used in the simulation (Fig. 2, bottom), where little structure is seen due to ergodization. A general discussion of this result is fairly involved, because it requires the analysis of field alignment of a scalar quantity $f$ in stochastic magnetic fields, i.e., $\boldsymbol{\nabla}_{\|} f=0$. However the following statements can be made:

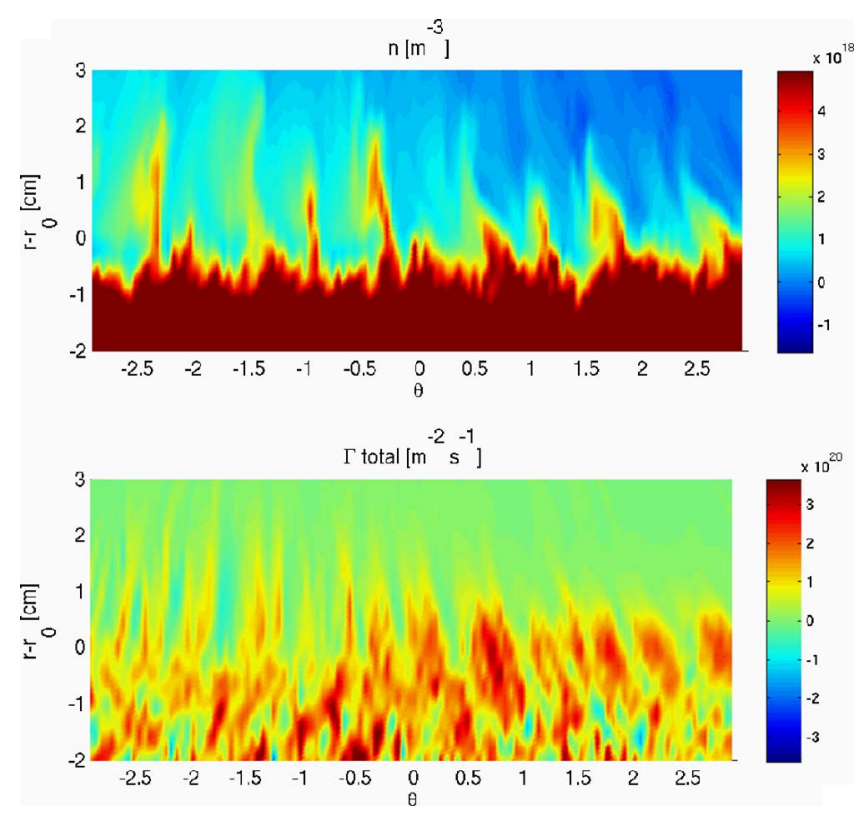

FIG. 6. (Color online) Top: Snapshot of the density profile $n$. Bottom: Time averaged local $E \times B$-flux $\bar{\Gamma}$. Both for the poloidal cut at $\varphi=0$. No magnetic perturbation included. 

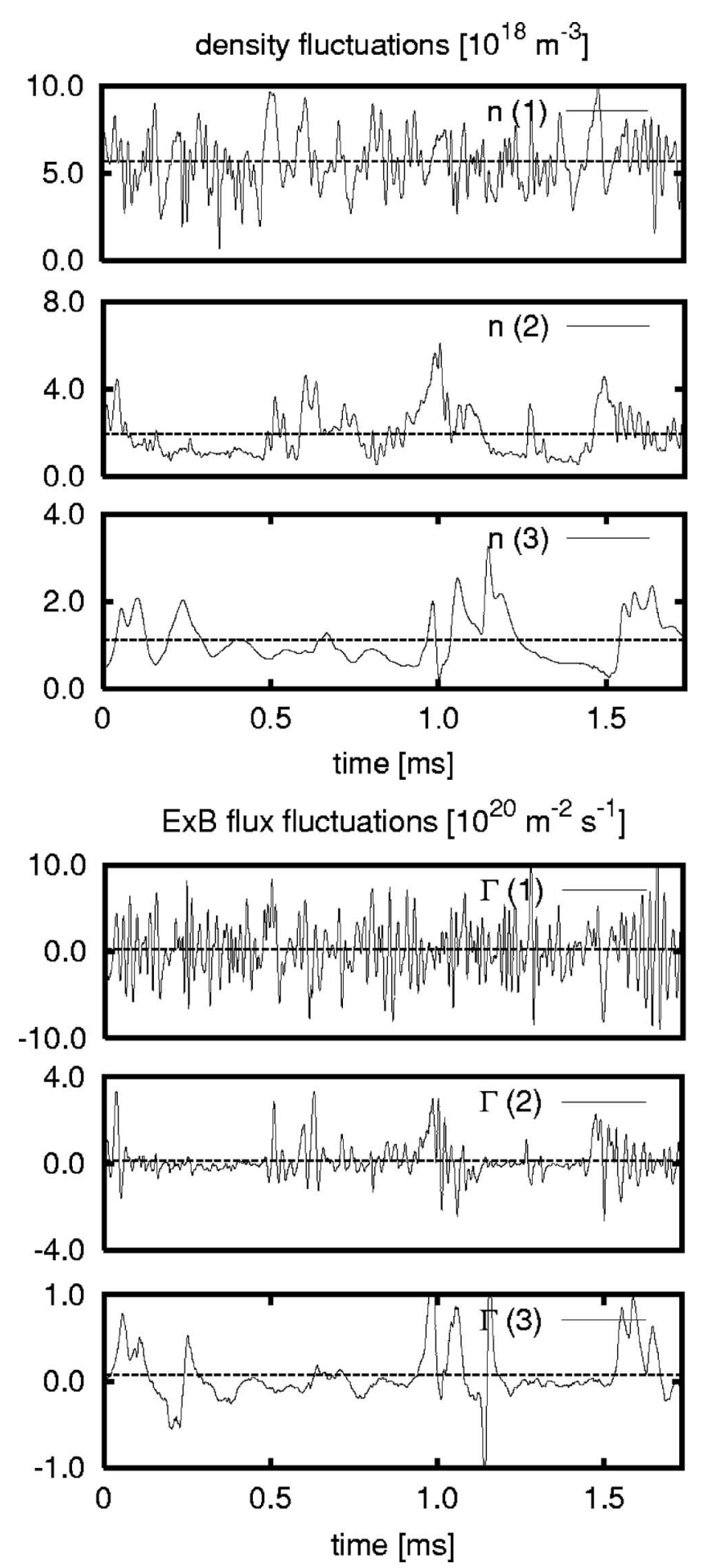

FIG. 7. Time traces of density $n$ and local $E \times B$-flux $\Gamma$ at three different probe positions, (1) $r=r_{0}-1 \mathrm{~cm}$, (2) $r=r_{0}$, (3) $r=r_{0}+1 \mathrm{~cm}$. No magnetic perturbation included. The horizontal lines indicate the temporal average.

(1) The Poincaré-plots of Fig. 2 represent the structure of the magnetic field and consequently, if a scalar quantity $f$ like the density would fulfill the equation $\nabla_{\|} f=0$ it would reveal all fine structured details of the Poincaré-plots.

(2) A perfect fulfillment of $\boldsymbol{\nabla}_{\|} f=0$ is not possible in the simulation due to lack of resolution of very fine structures and the action of a perpendicular dissipation, like e.g.,

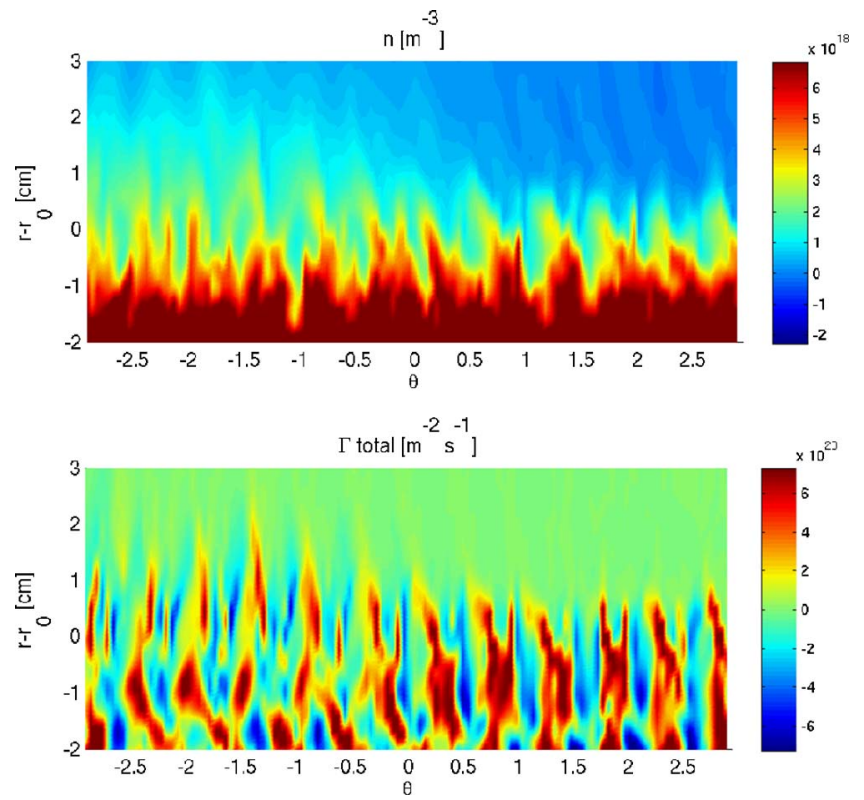

FIG. 8. (Color online) Top: Snapshot of the density profile $n$. Bottom: Time averaged local $E \times B$-flux $\bar{\Gamma}$. Both for the poloidal cut at $\varphi=0$. Magnetic perturbation included.

$\mu_{\perp} \nabla_{\perp}^{4} n$, causing a flattening of the profiles (cf. the decay in the spectra of Fig. 5 for high $k_{y}$ ).

Thus one can conclude that, e.g., $\nabla_{\|} / \bar{h} / \bar{h}$ is small (compared to perpendicular derivatives) but finite. This opinion is supported by Fig. 12, showing the points of intersection of field lines starting in the vicinity of the O-points of the 11/4 and 12/4 island chains. These points are marked according to the number of the toroidal turns made until the field lines return to the poloidal plane where they have been started. It can be seen that the intersection points after one and two toroidal turns cover regions which very much resemble residual islands similar to the patterns in Fig. 11. All together the island structures in Fig. 11 can be considered as a result of large parallel gradient lengths of the order $2 \pi R_{0}$ combined with a perpendicular dissipation inhibiting a fractal fine structure due to ergodization. The $E \times B$-flux shown in Fig. 11 is separated into its static contribution $\bar{n} \partial_{\eta} \bar{\phi}$ and the turbulent flow $\bar{\Gamma}-\bar{n} \partial_{\eta} \bar{\phi}$ and the results confirm that the alternating pattern already shown in Fig. 7 is originated in the static contribution and that the turbulent flux does not show a reversal in sign or pronounced symmetries.

\section{SUMMARY AND DISCUSSION}

The observations made in the simulations can be summarized as follows:

(1) Flux driven turbulence in the unperturbed edge-SOLregion allows density blobs to arise in the vicinity of the separatrix. These blobs can move about $1-2 \mathrm{~cm}$ into the SOL. Such blobs leave significant prints in the time trace of the local density fluctuations.

(2) For the unperturbed scenario the radial $E \times B$-transport also exhibits intermittency. However the 


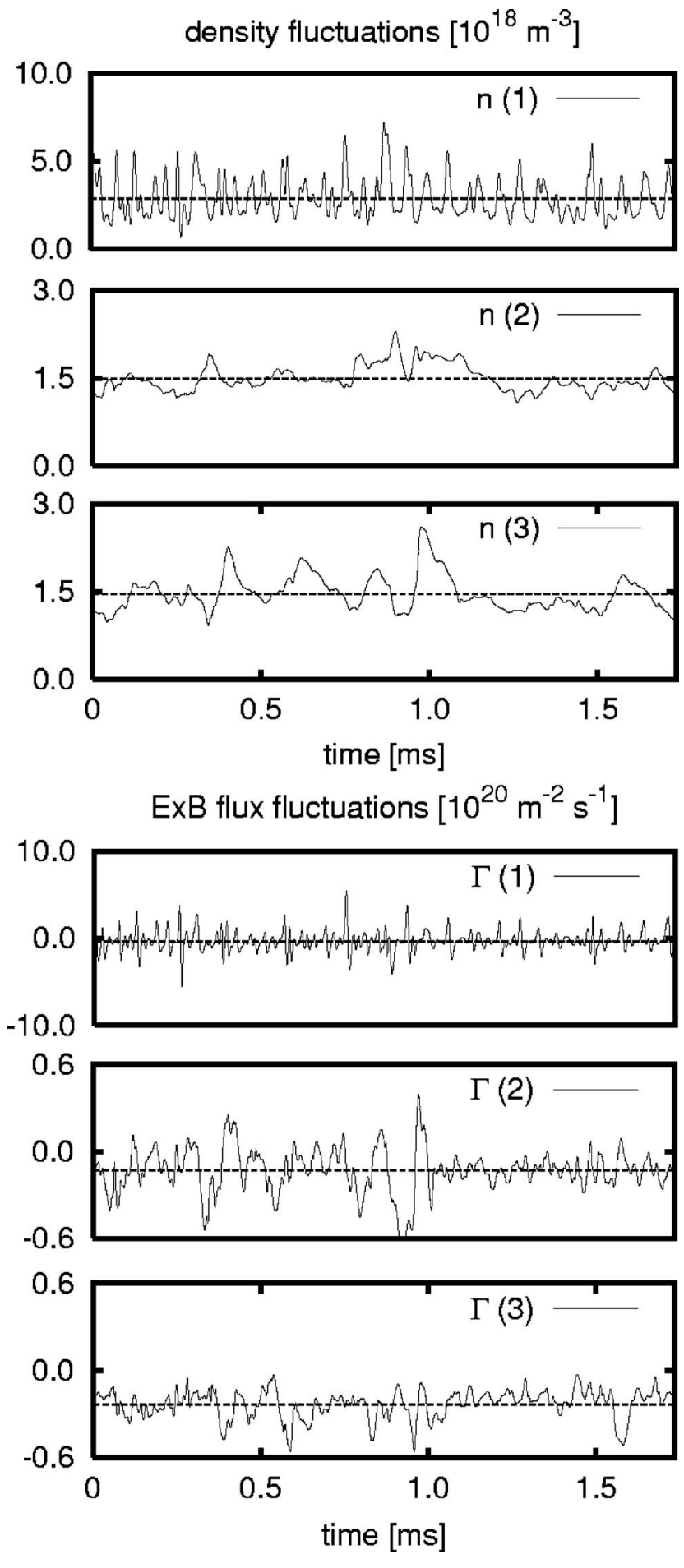

FIG. 9. Time traces of density $n$ and local $E \times B$-flux $\Gamma$ at three different probe positions, (1) $r=r_{0}-1 \mathrm{~cm}$, (2) $r=r_{0}$, (3) $r=r_{0}+1 \mathrm{~cm}$. Magnetic perturbation included. The horizontal lines indicate the temporal average.

time averaged $E \times B$-flow is relatively homogeneous and pointing radially outwards almost everywhere in the computational domain.

(3) The switch-on of a resonant magnetic perturbation flattens the flux-surface-averaged $(\sigma$ - $\eta$-averaged $)$ density profile. The net radial $E \times B$ is strongly reduced outside the LCFS.

(4) In the presence of magnetic perturbations the density blobs do not extend into the SOL anymore. Time traces of local density fluctuations show a strong amplitude reduction. It is to be noted that a suppression of density blobs due to magnetic perturbations has been observed recently at TEXTOR, ${ }^{5}$ and these findings seem to match the simulations presented here.

(5) Likewise the intermittency in the radial $E \times B$-flux is significantly reduced and additionally at certain locations its time averaged value is negative, i.e., pointing radially inwards. Actually the time averaged profile shows an alternating pattern of positive and negative signs in the flux giving a net radial transport of almost zero.

(6) The alternating pattern in the $E \times B$-flux is of static nature and it is found that the turbulent contribution is positive almost everywhere, but a factor 2-3 smaller than the peak values of the static contribution. In particular in the region around the O-points of the resonant 12/4-perturbation at the LCFS the turbulent $E \times B$-transport is almost vanishing.

(7) Even though the time averaged profiles of density and the electric potential resemble the symmetry of the perturbation field, i.e. $\bar{n} / \boldsymbol{\nabla}_{\|} \bar{n} \approx \bar{\phi} / \boldsymbol{\nabla}_{\|} \bar{\phi} \approx 2 \pi R_{0}$ (in particular a flattening of density around the O-points can be observed), the nonadiabatic part of the electron density $\bar{h}$ is clearly the quantity which is more strongly tied to the perturbation field.

All in all the simulations allow us to draw the conclusion that density blobs (and density fluctuations in general) can be suppressed by the action of a magnetic perturbation field and this is done basically via a restructuring of the equilibrium density and electric field and a suppression of turbulent $E \times B$-transport in the induced island chains.

Two questions are raised:

(a) What is the origin of the $E \times B$-pattern alternating in sign? The static pattern of $\bar{\Gamma}$ alternating in sign can be attributed to the result that $\bar{n}$ and $\bar{\phi}$ are field aligned to a large extent, which is a consequence of Eqs. (13) and (14) and the low resistivity $\hat{C}$ used in the simulations. However it is difficult to extract analytically more information about the symmetries of the static $E \times B$-flux just from the result that $\bar{n}$ and $\bar{\phi}$ show a certain field alignment without additional information on the detailed structure of the fields.

(b) What mechanism causes the reduction of turbulent fluctuations in density and $E \times B$-flux in the presence of a magnetic perturbation? A more detailed analysis of the time traces of the local dynamics of the density fluctuations show that the local turbulent drive by the radial density gradient is strongly reduced by magnetic perturbations due to the observed flattening of the (static) density profile around the O-points. But this does not give an explanation of the underlying mechanisms, i.e., whether the flattening is the cause or a consequence of the turbulent dynamics. Perhaps the same topological arguments used in Sec. V to explain the finite parallel gradient length of the static profiles, e.g. density, can be applied to a possible suppression of instabilities due to impeding $\boldsymbol{\nabla}_{\|} n=0$. Finally it should be mentioned that in experiments ${ }^{4}$ the turbulent $E \times B$-flux is not only reduced, it actually changes sign at least at certain locations.

Both issues need a further investigation of the details of 

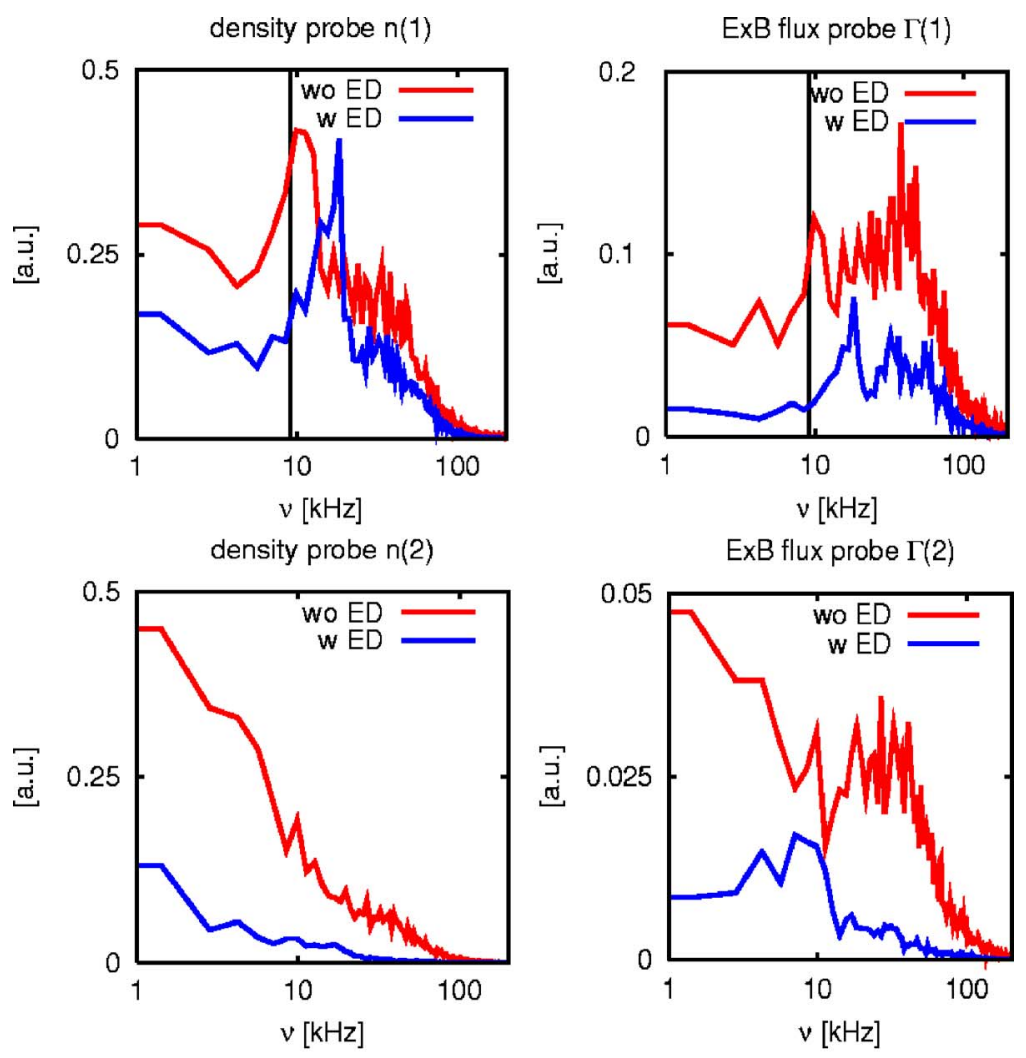

FIG. 10. (Color online) Frequency spectra of density $n$ and $E \times B$ flux $\Gamma$ for the three different probe positions, (top) $r=r_{0}-1 \mathrm{~cm}$, (middle) $r=r_{0}$, (bottom) $r=r_{0}+1 \mathrm{~cm}$. The vertical lines in the top graphs indicate the GAM frequency $(\approx 9.1 \mathrm{kHz})$.
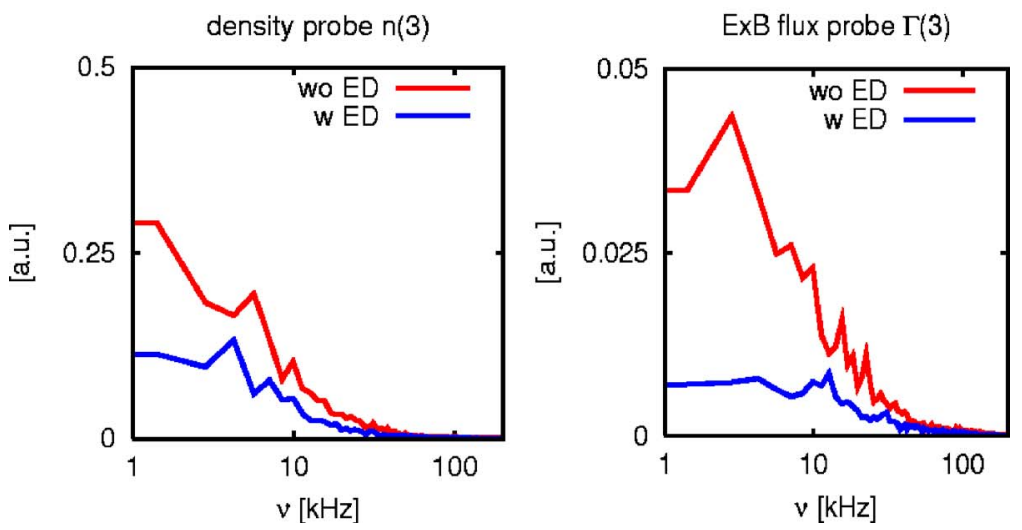

the turbulent dynamics and its interaction with static structures, e.g., the build up of zonal flows and the changes in energetics. This will be done in future work on this topic.

\section{CONCLUDING REMARKS}

The numerical results presented demonstrate that intermittent density fluctuations as observed in tokamak experiments are well captured by the drift fluid model used in this work in conjunction with the geometrical setup and the respective boundary conditions. The opinion that the numerical blob dynamics is closely related to the experiment is supported by the observation that the simulated turbulence exhibits a similar response to magnetic perturbations. It is to be noted that experience from previous numerical investigations based on models on different levels of complexity (2D/3D, electrostatic/electromagnetic, neglect/inclusion of temperature dynamics, drift-fluid/gyrofluid) give similar results on the characteristics of the density blobs appearing in edgeSOL-turbulence. This gives the hint that basic mechanisms are already captured by all of these approaches. The appearance of blobs seems to be quite a robust process due to the presence of a LCFS with adjoint regions of open and closed field lines and weak and strong parallel flows as well. It might be speculated that heat bursts show a similar behavior but of course this has to be proven by further numerical and analytical studies. A logical next step would be to employ a more extended model (like the GEM-model ${ }^{25}$ ), to see if the basic features described here survive when finite ion temperatures, temperature dynamics and more sophisticated limiter sheath models are included. Also this would allow us to study heat bursts in magnetic perturbation fields, which might be of potential relevance for the understanding of ELM-control.

\section{APPENDIX A: MODEL EQUILIBRIUM MAGNETIC FIELD AND FIELD ALIGNED COORDINATES}

To find an explicit form of the model Eqs. (1)-(5) it is necessary to prescribe an equilibrium magnetic field and to 

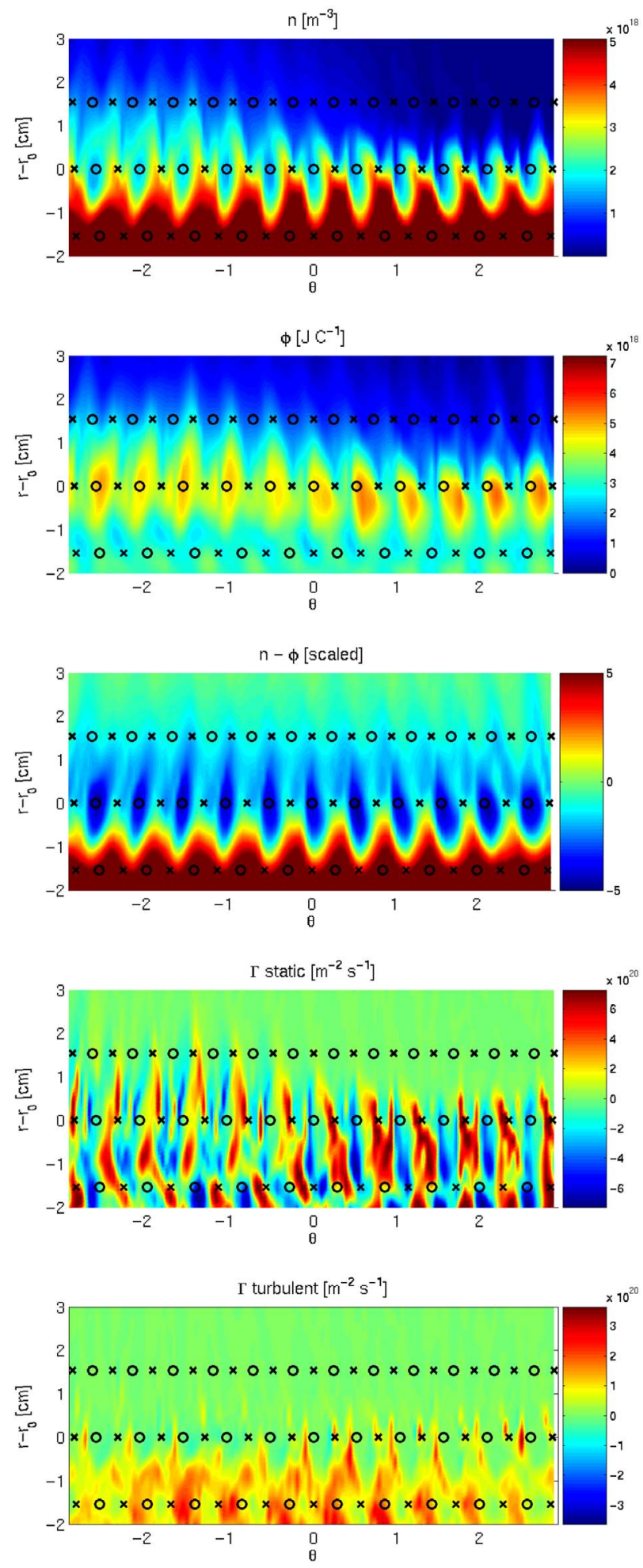

FIG. 11. (Color online) Time averaged profiles of density $n$, electric potential $\phi, h=n-\phi$, and the local $E \times B$-flux separated into its static and turbulent contribution (from top to bottom). The magnetic perturbation is included. The O-points and X-points of the perturbed magnetic field are labeled by "O" and "X," respectively.

define a suitable set of field aligned coordinates. Here we assume a toroidally axisymmetric magnetic equilibrium field consisting of nested flux surfaces. Such a field B can be prescribed by the transformation

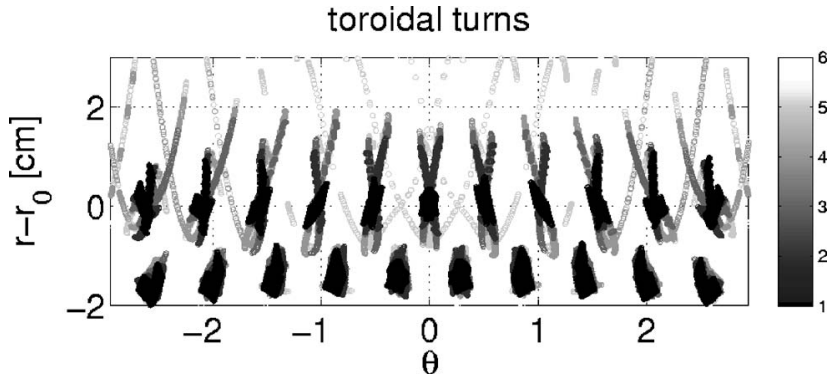

FIG. 12. Plot of the number of toroidal turns needed to move to the particular point starting from points in the vicinity of the O-points of the 11/4 and 12/4 island chains. The perturbation field is the one used in all simulations and corresponds to the Poincaré-plot of Fig. 2, bottom.

$$
x_{1}=R \cos \varphi ; \quad x_{2}=R \sin \varphi ; \quad x_{3}=Z,
$$

where $x_{1}, x_{2}$, and $x_{3}$ are the Cartesian coordinates and $R=R(r, \theta)$ and $Z=Z(r, \theta)$ are functions of the coordinates $r$ and $\theta$ in a way that closed flux surfaces are defined by $r=$ const., i.e., $r$ is a flux label. The coordinate $\varphi$ is the toroidal angle and the coordinate $\theta$ is the generalized poloidal angle. It is assumed that the coordinates $(r, \theta, \varphi)$ form a right-handed orthogonal system with Jacobian

$$
J=\mathbf{e}_{r} \cdot\left(\mathbf{e}_{\theta} \times \mathbf{e}_{\varphi}\right)=R\left(\frac{\partial R}{\partial \theta} \frac{\partial Z}{\partial r}-\frac{\partial R}{\partial r} \frac{\partial Z}{\partial \theta}\right)>0 .
$$

Due to the definition of $r$ as a flux label, the magnetic field is of the form

$$
\mathbf{B}=B^{\theta} \mathbf{e}_{\theta}+B^{\varphi} \mathbf{e}_{\varphi},
$$

where the toroidal field is prescribed by

$$
B^{\varphi}=\frac{1}{\sqrt{g_{\varphi \varphi}}} \frac{B_{0} R_{0}}{R}=\frac{B_{0} R_{0}}{R^{2}}
$$

corresponding to the $1 / R$-dependence of the toroidal field in a tokamak. The parameters $B_{0}$ and $R_{0}$ are constant, representing the field strength at the magnetic axis and its location. The requirement of a divergence free magnetic field gives

$$
B^{\theta}=\frac{B_{0} C}{J},
$$

where $C=C(r)$ is also a flux label. This completes the model for the magnetic field. Next field aligned coordinates are introduced by

$$
s=\theta ; \quad x=r ; \quad y=\int \frac{B^{\varphi}}{B^{\theta}} d \theta-\varphi
$$

with

$$
\nabla s \cdot(\nabla x \times \nabla y)=\nabla r \cdot(\nabla \theta \times \nabla \varphi)=J^{-1}
$$

and

$$
\mathbf{e}_{s}=\mathbf{e}_{\theta}+\frac{B^{\varphi}}{B^{\theta}} \mathbf{e}_{\varphi} ; \quad \mathbf{e}_{y}=-\mathbf{e}_{\varphi},
$$




$$
\mathbf{e}_{x}=\mathbf{e}_{r}+\int \frac{\partial}{\partial r}\left(\frac{B^{\varphi}}{B^{\theta}}\right) d \theta \mathbf{e}_{\varphi} .
$$

This coordinate transformation allows to represent our axisymmetric model field by the Clebsch-type form ${ }^{26}$

$$
\mathbf{B}=B_{0} C \nabla x \times \nabla y=B^{\theta} \mathbf{e}_{s} .
$$

The advantage of these coordinates is that due to the field alignment of the $s$-coordinate lines a convenient separation of the parallel and perpendicular dynamics with respect to $\mathbf{B}$ is possible. The disadvantage appearing now-compared to the system $(r, \theta, \varphi)$-is that in general the coordinates $(s, x, y)$ are not orthogonal. In particular the nonorthogonality of the $x$-coordinate and the $y$-coordinate causes severe difficulties in the numerical treatment of the perpendicular operators in the model equations considered in this paper. To overcome these problems the shifted metric procedure ${ }^{15}$ has been proposed and is now widely used in the numerical treatments of turbulence models for magnetized plasmas. ${ }^{13,10,27}$ Here we follow a similar approach by employing an analytical treatment introducing a third reference system labeled $(\sigma, \chi, \eta)$ where the inconvenient nonorthogonality is partially removed. These coordinates are defined by

$$
\sigma=\theta ; \quad \chi=r ; \quad \eta=\int \frac{B_{0}^{\varphi}}{B_{0}^{\theta}} d \theta-\varphi
$$

with $B_{0}^{\varphi} / B_{0}^{\theta}$ denoting the ratio of the covariant components of the magnetic field at a certain reference flux surface with $r=r_{0}$. Thus one obtains

$$
\nabla \sigma \cdot(\nabla \chi \times \nabla \eta)=\nabla s \cdot(\nabla x \times \nabla y)=J^{-1}
$$

and

$$
\mathbf{e}_{\sigma}=\mathbf{e}_{\theta}+\frac{B_{0}^{\varphi}}{B_{0}^{\theta}} \mathbf{e}_{\varphi} ; \quad \mathbf{e}_{\chi}=\mathbf{e}_{r} ; \quad \mathbf{e}_{\eta}=-\mathbf{e}_{\varphi}
$$

and

$$
\mathbf{B}=B^{\theta} \mathbf{e}_{\sigma}+\left(\frac{B_{0}^{\varphi}}{B_{0}^{\theta}}-\frac{B^{\varphi}}{B^{\theta}}\right) B^{\theta} \mathbf{e}_{\eta} .
$$

This means that this coordinate system is field aligned only at the reference flux surface.

In the following we confine ourselves to the following, widely used choice to specify $R$ and $Z$ in Eq. (A1),

$$
R=R_{0}+r \cos \theta ; \quad Z=-r \sin \theta
$$

representing nested, concentric circular flux surfaces. (Other choices are e.g., bipolar coordinates or a Miller equilibrium. $^{28,29}$ ) Thus the magnetic field $\mathbf{B}$ is given as

$$
\mathbf{B}=\frac{B_{0}}{q R} \mathbf{e}_{\theta}+\frac{B_{0} R_{0}}{R^{2}} \mathbf{e}_{\varphi},
$$

where $q=q(r)$ is the so-called pitch parameter, i.e., $C=r / q$. In this case the explicit forms of the field aligned coordinates defined in Eqs. (A6) and (A11) are

$$
s=\theta ; \quad x=r ; \quad y=q \theta^{*}-\varphi
$$

and

$$
\sigma=\theta ; \quad \chi=r ; \quad \eta=q_{0} \theta_{0}^{*}-\varphi,
$$

where

$$
\theta^{*}=\theta^{*}(r, \theta)=\frac{2}{\sqrt{1-\epsilon^{2}}} \arctan \left(\sqrt{\frac{1-\epsilon}{1+\epsilon}} \tan \frac{\theta}{2}\right)
$$

with $\epsilon=r / R_{0}, q_{0}=q\left(r_{0}\right)$, and $\theta_{0}^{*}=\theta^{*}\left(r_{0}, \theta\right)$.

Note that for the limit of high aspect ratio plasmas one obtains

$$
s=\theta ; \quad x=r ; \quad y=q \theta-\varphi+\mathcal{O}(\epsilon)
$$

and

$$
\sigma=\theta ; \quad \chi=r ; \quad \eta=q_{0} \theta-\varphi+\mathcal{O}(\epsilon)
$$

which resembles the definitions of field aligned coordinates in the cylindrical limit often used in the literature. ${ }^{23}$

\section{APPENDIX B: OPERATORS IN FLUTE APPROXIMATION}

To find the concrete form of the model equations in the coordinates $(s, x, y)$ and $(\sigma, \chi, \eta)$, defined in Eqs. (A17) and (A18), respectively, the relations found in Appendix A are used. The directional derivative along the magnetic field is simple due to the alignment of the coordinate $s$

$$
\begin{aligned}
\frac{\mathbf{B}}{B} \cdot \nabla f & =\frac{1}{q R_{0}} \frac{\partial f}{\partial s}+\mathcal{O}(\epsilon) \\
& =\frac{1}{q R_{0}}\left[\frac{\partial f}{\partial \sigma}+\left(q_{0}-q\right) \frac{\partial f}{\partial \eta}\right]+\mathcal{O}(\epsilon) .
\end{aligned}
$$

Next the perpendicular operators are approximated in the socalled flute approximation, i.e., the neglect of parallel compared to perpendicular derivatives, $\partial_{s} \ll \partial_{x}$, $\partial_{y}$. Thus it follows for the nonlinearities of the $E \times B$ advection and magnetic flutter

$$
\begin{aligned}
\frac{\mathbf{B}}{B^{2}} \cdot(\boldsymbol{\nabla} f \times \boldsymbol{\nabla} g) & =\frac{q}{r B_{0}}\left(\frac{\partial f}{\partial x} \frac{\partial g}{\partial y}-\frac{\partial f}{\partial y} \frac{\partial g}{\partial x}\right)+\mathcal{O}(\epsilon) \\
& =\frac{q}{r B_{0}}\left(\frac{\partial f}{\partial \chi} \frac{\partial g}{\partial \eta}-\frac{\partial f}{\partial \eta} \frac{\partial g}{\partial \chi}\right)+\mathcal{O}(\epsilon) .
\end{aligned}
$$

For the curvature term one finds

$$
\begin{aligned}
\boldsymbol{\nabla} \cdot\left(\frac{\mathbf{B} \times \boldsymbol{\nabla} f}{B^{2}}\right)= & -\frac{2}{B_{0} R_{0}}\left[\sin \theta \frac{\partial f}{\partial x}\right. \\
& \left.+(\cos \theta+\hat{s} \theta \sin \theta) \frac{q}{r} \frac{\partial f}{\partial y}\right]+\mathcal{O}(\epsilon) \\
= & -\frac{2}{B_{0} R_{0}}\left[\sin \theta \frac{\partial f}{\partial \chi}+\cos \theta \frac{q}{r} \frac{\partial f}{\partial \eta}\right]+\mathcal{O}(\epsilon) .
\end{aligned}
$$

The dimensionless quantity $\hat{s}=\partial \ln q / \partial \ln r$ denotes the magnetic shear. It has to be noted that the curvature term represents a real toroidal effect, even in the limit $\epsilon \rightarrow 0$, which would not appear if one considers an equilibrium field with the symmetry of a periodic cylinder! Finally the operator $\nabla_{\perp}^{2}$ 
is evaluated in slab approximation (i.e., neglect of Christoffel symbols) as

$$
\boldsymbol{\nabla}_{\perp}^{2}=g^{x x} \frac{\partial^{2}}{\partial x^{2}}+2 g^{x y} \frac{\partial^{2}}{\partial x \partial y}+g^{y y} \frac{\partial^{2}}{\partial y^{2}}
$$

which gives

$$
\begin{aligned}
\nabla_{\perp}^{2} f & =\frac{\partial^{2} f}{\partial x^{2}}+\frac{2 \hat{s} q}{r} \frac{\partial^{2} f}{\partial x \partial y}+\frac{q^{2}\left(1+\hat{s}^{2} \theta^{2}\right)}{r^{2}} \frac{\partial^{2} f}{\partial y^{2}}+\mathcal{O}(\epsilon) \\
& =\frac{\partial^{2} f}{\partial \chi^{2}}+\frac{q^{2}}{r^{2}} \frac{\partial^{2} f}{\partial \eta^{2}}+\mathcal{O}(\epsilon) .
\end{aligned}
$$

In the derivation of the expressions (B2), (B3), and (B5) the relations

$$
\frac{\partial}{\partial x}=\frac{\partial}{\partial \chi}-\frac{\hat{s} \theta q}{r} \frac{\partial}{\partial \eta}+\mathcal{O}(\epsilon) ; \quad \frac{\partial}{\partial y}=\frac{\partial}{\partial \eta}
$$

have been used. The advantage of the system $(s, x, y)$ is in the easy separation of the anisotropic turbulent dynamics in parallel and perpendicular parts and in the simple expression for the parallel derivative. The subsequent transformation into the system $(\sigma, \chi, \eta)$ allows a considerable simplification of the perpendicular operators with respect to numerical purposes because $g^{\chi \eta}=0$, whereas $g^{x y} \neq 0$.

\section{APPENDIX C: SCALING AND PARAMETERS}

For the sake of better readability, more convenient numerical implementation and to point out the basic parameters guiding the dynamics of the turbulence model used in this paper, a proper scaling is introduced to obtain the dimensionless form Eqs. (11)-(15). This scaling is guided by

$$
\begin{aligned}
& \chi \rightarrow r_{0}+\rho_{s} \chi ; \quad \eta \rightarrow \frac{q_{0} \rho_{s}}{r_{0}} \eta, \\
& \phi \rightarrow \frac{\rho_{s} T_{e}}{e L_{\perp}} \phi ; \quad n \rightarrow \frac{\rho_{s} n_{0}}{L_{\perp}} n, \\
& A \rightarrow \frac{\rho_{s}^{2} q_{0} R_{0} \beta B_{0}}{L_{\perp}^{2}} A ; \quad u \rightarrow \frac{\rho_{s} q_{0} R_{0} c_{s}}{L_{\perp}^{2}} u, \\
& J \rightarrow \frac{\rho_{s} q_{0} R_{0} e n_{0} c_{s}}{L_{\perp}^{2}} J ; \quad t \rightarrow \frac{L_{\perp}}{c_{s}} t, \\
& \nabla_{\perp}^{2} \rightarrow \frac{\nabla_{\perp}^{2}}{\rho_{s}^{2}} ; \quad \nabla_{\|} \rightarrow \frac{\nabla_{\|}}{q_{0} R_{0}}, \\
& \mathcal{K} \rightarrow \frac{\mathcal{K}}{B_{0} L_{\perp} \rho_{s}} ; \quad B \rightarrow B_{0} B,
\end{aligned}
$$

where

$$
\beta=\frac{\mu_{0} n_{0} T_{e}}{B_{0}^{2}} ; \quad \rho_{s}=\frac{c_{s} m_{i}}{e B_{0}},
$$

$$
c_{s}=\sqrt{\frac{T_{e}}{m_{i}}} ; \quad \nu_{e}=\frac{e^{4} n_{0} \ln \Lambda}{3(2 \pi)^{3 / 2} \epsilon_{0}^{2} m_{e}^{1 / 2} T_{e}^{3 / 2}}
$$

such that the basic parameters of the model are given by

$$
\begin{aligned}
& \hat{\epsilon}=\left(\frac{q_{0} R_{0}}{L_{\perp}}\right)^{2} ; \quad \hat{\beta}=\hat{\epsilon} \beta ; \quad \hat{\mu}=\hat{\epsilon} \frac{m_{e}}{m_{i}}, \\
& \hat{C}=\hat{\mu} \frac{L_{\perp}}{c_{s}} \frac{\nu_{e}}{1.96} ; \quad \omega_{B}=2 \frac{L_{\perp}}{R_{0}} .
\end{aligned}
$$

The quantities $n_{0}$ and $q_{0}$ represent a density and pitch parameter at the reference radius $r_{0}$. The parameter $L_{\perp}$ denotes a typical background radial gradient length.

${ }^{1}$ T. E. Evans, R. A. Moyer, K. H. Burrell, M. E. Fenstermacher, I. Joseph, A. W. Leonard, T. H. Osborne, G. D. Porter, M. J. Schaffer, P. B. Snyder, P. R. Thomas, J. G. Watkins, and W. P. West, Nat. Phys. 2, 419 (2006).

${ }^{2}$ T. E. Evans, K. H. Burrell, M. E. Fenstermacher, R. A. Moyer, T. H. Osborne, M. J. Schaffer, W. P. West, L. W. Yan, J. A. Boedo, E. J. Doyle, G. L. Jackson, I. Joseph, C. J. Lasnier, A. W. Leonard, T. L. Rhodes, P. R. Thomas, J. G. Watkins, and L. Zeng, Phys. Plasmas 13, 056121 (2006).

${ }^{3}$ Y. Liang, H. R. Koslowski, P. R. Thomas, E. Nardon, B. Alper, P. Andrew, Y. Andrew, G. Arnoux, Y. Baranov, M. Bécoulet, M. Beurskens, T. Biewer, M. Bigi, K. Crombe, E. De La Luna, P. de Vries, W. Fundamenski, S. Gerasimov, C. Giroud, M. P. Gryaznevich, N. Hawkes, S. Hotchin, D. Howell, S. Jachmich, V. Kiptily, L. Moreira, V. Parail, S. D. Pinches, E. Rachlew, and O. Zimmermann, Phys. Rev. Lett. 98, 265004 (2007).

${ }^{4}$ Y. Xu, R. R. Weynants, S. Jachmich, M. Van Schoor, M. Vergote, P. Peleman, M. W. Jakubowski, M. Mitri, D. Reiser, B. Unterberg, and K. H. Finken, and the TEXTOR team, Phys. Rev. Lett. 97, 165003 (2006).

${ }^{5} \mathrm{Y}$. Xu (private communications, 2007) (to be presented at the 34th EPS conference).

${ }^{6}$ Y. Sarazin and Ph. Ghendrih, Phys. Plasmas 5, 4046 (1998).

${ }^{7}$ O. E. Garcia, V. Naulin, A. H. Nielsen, and J. Juul Rasmussen, Phys. Rev. Lett. 92, 165003 (2004).

${ }^{8}$ O. E. Garcia, V. Naulin, A. H. Nielsen, and J. Juul Rasmussen, Phys. Plasmas 12, 062309 (2005).

${ }^{9}$ N. Bisai, A. Das, S. Deshpande, R. Jha, P. Kaw, A. Sen, and R. Singh, Phys. Plasmas 12, 072520 (2005).

${ }^{10}$ T. T. Ribeiro and B. Scott, Plasma Phys. Controlled Fusion 47, 1657 (2005).

${ }^{11}$ D. Reiser and B. Scott, Phys. Plasmas 12, 122308 (2005).

${ }^{12}$ P. Beyer, X. Garbet, and P. Ghendrih, Phys. Plasmas 5, 4271 (1998).

${ }^{13}$ V. Naulin, Phys. Plasmas 10, 4016 (2003).

${ }^{14}$ B. D. Scott, New J. Phys. 4, 52 (2002).

${ }^{15}$ B. Scott, Phys. Plasmas 8, 447 (2001).

${ }^{16}$ S. I. Braginskii, Rev. Math. Phys. 1, 205 (1965).

${ }^{17}$ M. Wakatani and A. Hasegawa, Phys. Fluids 27, 611 (1984).

${ }^{18}$ B. D. Scott, Phys. Fluids B 4, 2468 (1992).

${ }^{19}$ F. L. Hinton and C. W. HortonJr., Phys. Fluids 14, 116 (1971).

${ }^{20}$ B. D. Scott, Plasma Phys. Controlled Fusion 39, 1635 (1997).

${ }^{21}$ G. E. Karniadakis, M. Israeli, and S. A. Orszag, J. Comput. Phys. 97, 414 (1991).

${ }^{22}$ A. Arakawa, J. Comput. Phys. 135, 103 (1997).

${ }^{23}$ M. A. Beer, S. C. Cowley, and G. W. Hammett, Phys. Plasmas 2, 2687 (1995).

${ }^{24}$ P. H. Diamond, S. H. Itoh, K. Itoh, and T. S. Hahm,Plasma Phys. Controlled Fusion 47, R35 (2005).

${ }^{25}$ B. Scott, Phys. Plasmas 12, 102307 (2005).

${ }^{26}$ W. D. D'haeseleer, W. N. G. Hitchon, J. D. Callen, and J. L. Shohet, Flux Coordinates and Magnetic Field Structure (Springer-Verlag, Berlin, 1990), p. 100.

${ }^{27}$ B. Scott, Plasma Phys. Controlled Fusion 45, A385 (2003).

${ }^{28}$ G. A. Korn and T. M. Korn, Mathematical Handbook for Scientists and Engineers (McGraw-Hill, New York, 1961), p. 182.

${ }^{29}$ R. L. Miller, M. S. Chu, J. M. Greene, Y. R. Lin-Liu, and R. E. Waltz, Phys. Plasmas 5, 973 (1998). 